\title{
The sustained influence of prior experience induced by social observation on placebo and nocebo responses
}

This article was published in the following Dove Press journal: Journal of Pain Research

\author{
Huijuan Zhang' \\ Lili Zhou ${ }^{2,3}$ \\ Hua Wei' \\ Xuejing $\mathrm{Lu}^{2,3}$ \\ $\mathrm{Li} \mathrm{Hu}{ }^{1-3}$ \\ 'Faculty of Psychology, Southwest \\ University, Chongqing, China; ${ }^{2} \mathrm{CAS}$ \\ Key Laboratory of Mental Health, \\ Institute of Psychology, Beijing, China; \\ ${ }^{3}$ Department of Psychology, University \\ of Chinese Academy of Sciences, \\ Beijing, China
}

Background: Social observation is one of the main ways to gain experience. Similar to firstperson experience, observational experience affects the effectiveness of subsequent treatments. Yet, it is still undetermined whether the influence of social observation on placebo and nocebo effects to subsequent treatments remains even if related experience occurred a few days ago.

Methods: Eighty-two participants were recruited and each of them was randomly assigned to one of the four experimental groups acquiring first-person or observational experience, which was either effective or ineffective. For the first-person groups, participants were presented with pain cues paired with pain stimuli in person. In the effective condition, low pain cues were paired with low pain stimuli, and high pain cues were paired with high pain stimuli. In contrast, the associations between cues and pain stimuli were not established in the ineffective condition. Similarly, for the observational groups, participants received effective/ineffective treatment through observation. Five or six days later, all participants underwent a conditioning phase followed by a test phase composed of two tests, where participants were asked to report their perceived pain. Results: Placebo and nocebo responses to subsequent treatments can be affected by prior experience gained several days ago regardless of acquisition ways, and both placebo and nocebo responses in the effective condition were significantly larger than those in the ineffective condition. Furthermore, once placebo and nocebo effects were elicited, the latter was more persistent, while the former was more likely to diminish.

Conclusion: First-person and observational experience obtained a few days ago could affect the following treatments, which advance our understanding of the crucial and sustained influence of social observation on placebo analgesia and nocebo hyperalgesia, and provide insights into clinical applications.

Keywords: social observation, prior experience, placebo analgesia, nocebo hyperalgesia, conditioning, expectancy

\section{Introduction}

Learning plays an important role in the induction of placebo/nocebo responses via firstperson experience and social observation, ${ }^{1,2}$ two main acquisition ways that shape prior experience thereby altering our perceived effectiveness of treatments. ${ }^{3}$ Numerous clinical observations have demonstrated the pivotal role of prior first-person experience in placebo analgesia and nocebo hyperalgesia, ${ }^{4-11}$ that is, robust placebo/nocebo responses can be induced after repeated exposure to positive/negative treatments in person. ${ }^{4-11}$ Similar situations can be observed in laboratory settings, where prior first-person experience of positive treatments induces strong placebo responses; negative treatments, on the other hand, may reduce placebo responses and even lead to nocebo responses. ${ }^{8,12,13}$
Correspondence: Li Hu; Xuejing Lu Key Laboratory of Mental Health, Institute of Psychology, Chinese Academy of Sciences, 16 Lincui Road, Chaoyang District, Beijing I00I0I, China

Tel +86 I80 84053555

Fax +86 10 84249369

Email huli@psych.ac.cn; luxj@psych.ac.cn 
Similar to first-person experience, social observation can also generate strong placebo analgesia and nocebo hyperalgesia. ${ }^{14-18}$ However, it is still unclear whether the placebo/nocebo responses generated by social observation are comparable to those generated by first-person experience, in terms of their sustainability. If the effect of social observation can sustain, it would be of great significance in clinical applications, as patients could obtain long-term benefits for their rehabilitation from acquiring positive experience and avoiding negative experience through social observation.

In addition to the sustainability of the influence of prior experience on placebo/nocebo responses to subsequent treatments, there is less attention to the persistence of placebo/ nocebo responses. Previous studies based on first-person experience showed that 1) the persistence of placebo and nocebo responses can be influenced by the strength of learning (ie, the number of conditioning trials), ${ }^{19}$ and 2) placebo analgesia established under continuous reinforcement rather than partial reinforcement can be extinguished during the test phase, ${ }^{20}$ whereas nocebo hyperalgesia, once established, seems difficult to disappear irrespective of the conditioning schedules (continuous reinforcement and partial reinforcement). ${ }^{21}$ In contrast, the persistence of placebo and nocebo responses induced by social observation has not been well examined.

In the present study, we aimed at examining the sustained effect of prior experience induced by social observation on placebo/nocebo responses to subsequent treatments. Based on previous studies, ${ }^{3-21}$ we hypothesized that, as the prior experience in person, the experience induced by social observation would have sustained influence on placebo/nocebo responses. In other words, the placebo/nocebo responses in an effective prior experience condition would be larger than those in an ineffective prior experience condition. Furthermore, compared with nocebo hyperalgesia, placebo analgesia would be more likely to be extinguished. To test these hypotheses, we first investigated the sustainability of the effect of either effective or ineffective prior experience, acquired a period of time ago through first-person experience or social observation, on placebo and nocebo responses to subsequent treatments. Second, we included two successive tests to explore the difference between nocebo and placebo responses generated by the two experience acquisition ways, in terms of their persistence.

\section{Materials and methods Participants}

To rule out the possible confounding factors of handedness and gender on pain perception and placebo/nocebo effects, ${ }^{17,22}$ only right-handed healthy female volunteers were recruited in this study ( $\mathrm{N}=82$; mean age $20.73 \pm 1.56$ years). According to self-reports, none had history of major medical or psychiatric illness, or alcohol or drug abuse. All volunteers were informed that the aim of the present study was to investigate "the individual difference in pain perception and its psychological mechanism," while the true purpose of the study was not revealed until the end of the experiment. The study was approved by Ethics Committee of Southwest University, and informed consent forms were signed by participants before the experiment.

\section{Nociceptive stimuli}

The electrical nociceptive stimuli were delivered using a constant-current stimulator (model DS7A, Digitimer, UK), with three stainless-steel concentric bipolar needle electrodes. Each electrode consisted of a needle cathode (length: 0.1 $\mathrm{mm}$, Ø: $0.2 \mathrm{~mm}$ ) surrounded by a cylindrical anode (Ø: 1.4 $\mathrm{mm}$ ), which has been proved to preferentially activate $\mathrm{A} \delta$ nociceptive fibers in the superficial skin layers. ${ }^{23,24}$ All three electrodes were pasted on the inner side of participants' left forearm according to an equilateral triangle shape. Each stimulus consisted of several (ranging from 1 to 20, varied within subjects to elicit graded intensity of pain perception) rapidly succeeding constant-current, square-wave pulses (0.5-ms duration for each pulse), which were presented at $50 \mathrm{~Hz}$, with an intensity ranging from 0.8 to $3.0 \mathrm{~mA}$ (varied between subjects to account for the individual difference of pain sensitivity).

The method of limits (an ascending series of stimuli in steps of $1 \mathrm{~mA}$ were delivered starting from subtactile threshold until pain sensation was induced ${ }^{25}$ ) was applied to identify the stimulus intensity and the number of pulses for each participant that would elicit a low pain sensation at $\sim 2$ rating, moderate pain sensation at $\sim 5$ rating, and high pain sensation at $\sim 8$ rating on a self-report 11-point Numeric Rating Scale (NRS) $\left(0=\right.$ no sensation, $10=$ unbearable pain) ${ }^{26}$ Participants were familiarized with the electrical stimuli before the treatment phase and the conditioning phase.

\section{Experimental procedure}

A random, single-blinded, between-subject experimental design was adopted in the present study. As shown in Figure 1, the experiment consisted of three phases (ie, treatment phase, conditioning phase, and test phase) on two separate days. All participants were tested by a female experimenter, who wore a white coat and had received systematic training about the experimental procedure before the formal experiment. 


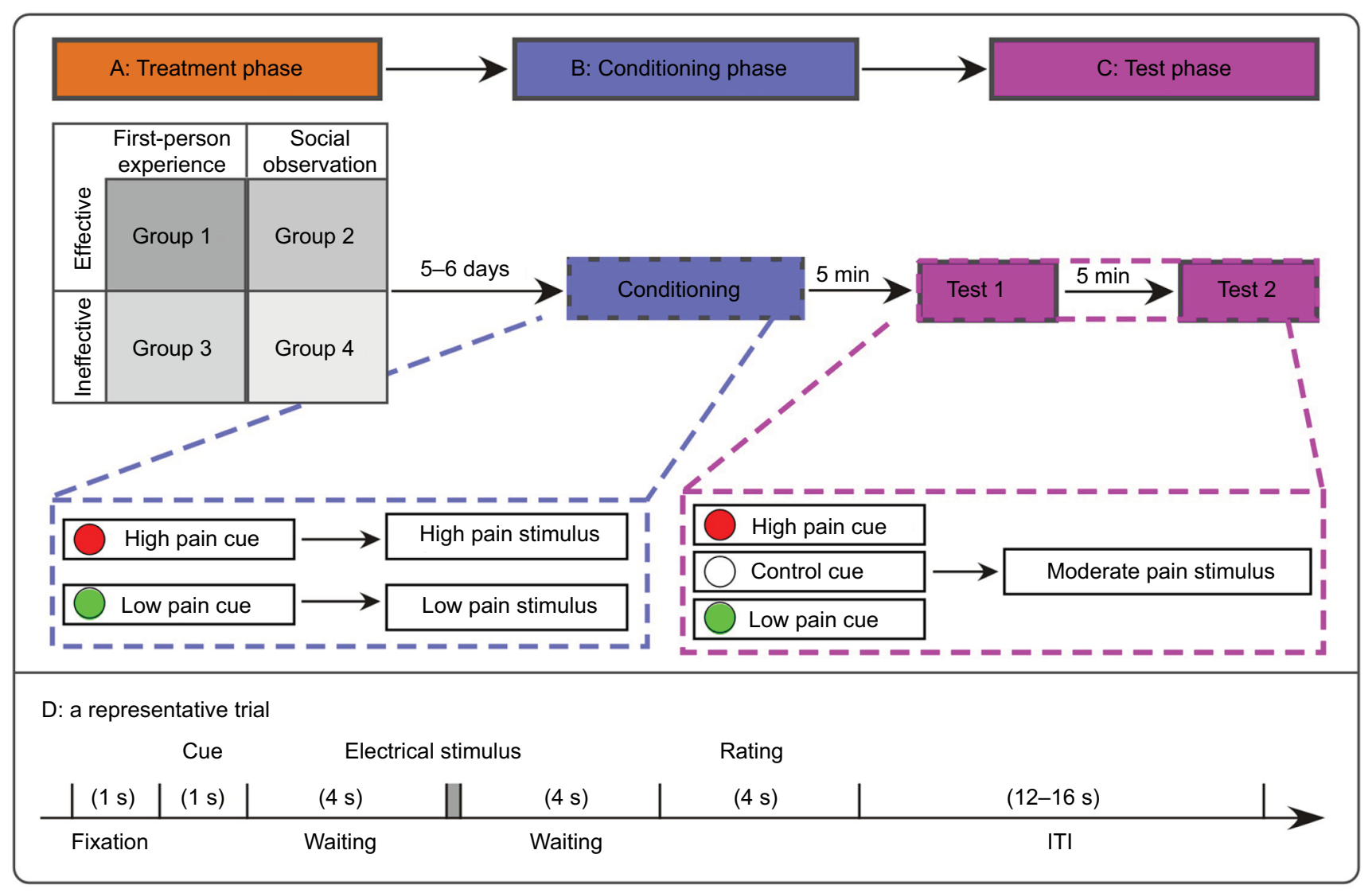

Figure I The experimental design.

Notes: The experimental procedure consisted of three phases on two separate days, including treatment phase, conditioning phase, and test phase (tests I and 2). (A) Treatment phase. First-person experience refers to receiving treatment in person, and observation experience refers to observing a treatment. For the effective condition, visual cues with different colors were paired with nociceptive stimuli with different intensities (low pain cues were paired with low pain stimuli, and high pain cues were paired with high pain stimuli). In contrast, for the ineffective condition, low pain cues were paired with low pain stimuli in one half of the trials, but paired with high pain stimuli in the other half of the trials (the same for high pain cues). In this phase, participants were randomly assigned to four experimental groups divided by "Acquisition Way" (firstperson/observation experience) and "Effectiveness" (effective/ineffective). "Effective" means the associations between cues and pain stimuli were established. When the low pain cues appeared, participants perceived an analgesic effect of the device (the metal ring). When the high pain cues appeared, participants perceived a hyperalgesic effect of the device. "Ineffective" means the associations between cues and pain stimuli were not established, and participants could not perceive analgesic effect/hyperalgesic effect. Group I: effective first-person experience group; Group 2: effective observation experience group; Group 3: ineffective first-person experience group; Group 4: ineffective observation experience group. (B) Conditioning phase. After 5-6 days, all participants underwent the same conditioning procedure. Visual cues with different colors were paired with nociceptive stimuli with different intensities, that is, low pain cues were consistently paired with low pain stimuli, and high pain cues were consistently paired with high pain stimuli. (C) Test phase. The test phase was composed of two tests (tests I and 2), with a 5-min interval. All visual cues (low pain, control, and high pain) were followed by moderate pain stimuli. (D) A representative trial, which was used in all three phases.

Abbreviation: ITI, inter-trial interval.

\section{Treatment phase}

According to the acquisition way (experience in person vs through observation) and effectiveness (effective vs ineffective) of different prior experience, participants were randomly assigned to four experimental groups (Group 1: effective first-person experience group; Group 2: effective observation experience group; Group 3: ineffective first-person experience group; Group 4: ineffective observation experience group); (Table 1) during the treatment phase (Figure 1).

Participants in each group undertook a total of 36 trials, which started with a 1-s white fixation cross centered on the monitor with black background in the front of participants at a distance of $\sim 50 \mathrm{~cm}$, followed by a visual cue (either a red or green dot) lasting for $1 \mathrm{~s}$. For half of the participants, the red dot matched with high pain stimuli (ie, high pain cue), and the green dot matched with low pain stimuli (ie, low pain cue), and vice versa for the other half of the participants, to rule out the possible confounding factor of the color of the visual cue. ${ }^{16,27}$ After a 4-s blank, a nociceptive stimulus was delivered to participants' left volar forearm. After another 4-s blank, participants were required to verbally rate the pain intensity on the 11-point NRS. The inter-trial interval (ITI) varied randomly between 12 and $16 \mathrm{~s}$. The whole session lasted for $\sim 20 \mathrm{~min}$.

More specifically, participants in Group 1 received an effective placebo treatment in person, where half of the trials presented high pain stimuli ( $\sim 8$ rating on an 11-point selfreport NRS) following the high pain cue, and the remaining 
Table I Characteristics of participants in each experimental group

\begin{tabular}{|c|c|c|c|c|c|c|c|}
\hline Characteristics & $\begin{array}{l}\text { Effective first- } \\
\text { person experience } \\
(\mathbf{N}=22)\end{array}$ & $\begin{array}{l}\text { Effective } \\
\text { observation } \\
\text { experience }(\mathrm{N}=\mathbf{2} \mathrm{I})\end{array}$ & $\begin{array}{l}\text { Ineffective first- } \\
\text { person experience } \\
(\mathrm{N}=20)\end{array}$ & $\begin{array}{l}\text { Ineffective } \\
\text { observation } \\
\text { experience }(N=19)\end{array}$ & $F(3,78)$ & $P$ & $\eta_{p}^{2}$ \\
\hline Age (years) & $21.23 \pm 1.63$ & $20.52 \pm 1.50$ & $20.50 \pm 1.64$ & $20.63 \pm 1.46$ & 1.034 & 0.382 & 0.038 \\
\hline PCS & $18.55 \pm 10.47$ & $16.19 \pm 9.92$ & $22.35 \pm \mid 2.31$ & $|7.89 \pm| 1.05$ & 1.14 & 0.336 & 0.042 \\
\hline STAI-S & $38.18 \pm 10.82$ & $38.90 \pm 7.60$ & $40.35 \pm 10.20$ & $39.89 \pm 9.24$ & 0.21 & 0.884 & 0.008 \\
\hline STAI-T & $43.27 \pm 8.82$ & $42.29 \pm 9.13$ & $43.30 \pm 8.82$ & $41.28 \pm 9.99$ & 0.08 & 0.971 & 0.003 \\
\hline IRI & $55.95 \pm 9.30$ & $56.57 \pm 8.33$ & $55.85 \pm 7.56$ & $59.21 \pm 7.15$ & 0.724 & $0.54 I$ & 0.027 \\
\hline
\end{tabular}

Note: Data are expressed as mean \pm SD.

Abbreviations: IRI, Interpersonal Reactivity Index; PCS, Pain Catastrophizing Scale; STAI-S, State Subscale of State-Trait Anxiety Inventory; STAI-T, Trait Subscale of StateTrait Anxiety Inventory.

half delivered low pain stimuli ( 2 rating on an 11-point self-report NRS) following the low pain cue. A metal ring (a sham device) served as the placebo and was attached to the thumb of participants' left hands. Participants were informed that the intensity of nociceptive stimuli was constant. The low pain cues would trigger the device to deliver a sub-threshold stimulus that induced an analgesic effect, whereas the high pain cues would trigger the device to induce a hyperalgesia effect. As a matter of fact, however, the stimulus intensities associated to the low pain cues were surreptitiously decreased, and the stimulus intensities paired with the high pain cues were surreptitiously increased. Therefore, after receiving the effective placebo treatment, participants would perceive an analgesic effect of the device (the metal ring), when the low pain cues appear, and a hyperalgesia effect of the device, when the high pain cues appear.

In Group 2, participants were asked to watch a self-made video where a 24-year-old male demonstrator was receiving an effective placebo treatment. The video was recorded in the same room, in which the experiment took place. The demonstrator was carefully trained to simulate the response to an effective placebo treatment prior to the video recording. The video showed that the demonstrator was sitting in front of a computer screen, with an electrode applied to the left volar forearm and a metal ring attached to the left thumb. The demonstrator received a sequence of pain stimuli following visual cues identical to Group 1 and was required to rate the stimulus intensity. Notably, all ratings of pain stimuli were predefined, that is, the average ratings to the stimuli following low pain cues and high pain cues were 2 and 8 on the NRS, respectively. To ensure the participants focused on the video, they were required to memorize the number of visual cues paired with a rating of $>5$ and that of $<5$, respectively. ${ }^{15}$ After the observation, participants were told that they would undergo an experimental session similar to the demonstrator's experience 5-6 days later.
For Group 3, to ensure that the participants experienced an ineffective treatment (ie, the associations between cues and pain stimuli cannot be established, that is, participants would not perceive an analgesic effect or a hyperalgesic effect of the device) in person, the high pain cues were followed by 9 high pain stimuli and 9 low pain stimuli, while the low pain cues matched with another 9 high pain stimuli and 9 low pain stimuli. All stimuli were delivered to participants in a pseudorandom sequence. Participants wore the sham device and got instructions identical to those in Group 1.

Participants in Group 4 were asked to watch a different self-made video, in which the demonstrator received the same ineffective placebo treatment as Group 3. Notably, all ratings of pain stimuli were also predefined, that is, the average ratings to the low pain stimuli and high pain stimuli were 2 and 8 on NRS, respectively. In contrast, the average ratings to the low pain cues and high pain cues were identical. The rest procedure was identical to that in Group 2.

\section{Conditioning phase}

After 5-6 days, all participants wearing the sham device underwent the same experimental procedure during conditioning phase. Specifically, half of the trials presented high pain stimuli following high pain cues, and the other half delivered low pain stimuli following low pain cues. The rest procedure of this phase was the same as Group 1 in treatment phase. This whole phase lasted for about $20 \mathrm{~min}$. With this phase, we could assess the influence of prior experience, either induced by social observation or in person, on the newly established placebo and nocebo effects to subsequent treatment.

\section{Test phase}

To verify the persistence of the analgesia and hyperalgesia effects, the test phase was composed of two identical tests (ie, test 1 and test 2), with a 5-min interval. In addition to 12 low 
pain cues and 12 high pain cues, 12 control cues (ie, white dot) were included in each test, and all visual cues were followed by moderate pain stimuli with $\mathrm{a} \sim 5$ rating on the NRS. All trials were administered in a pseudorandom sequence. Participants were informed that low pain cues anticipated an analgesic effect and high pain cues anticipated a hyperalgesia effect of the device. In addition, when control cues appeared, participants were informed that the device would be turned off and there was no modulational effect on the perceived pain intensity. The test phase lasted for $\sim 45 \mathrm{~min}$.

\section{Questionnaires}

All participants were required to complete three questionnaires before conditioning phase, which included the Chinese versions of the Pain Catastrophizing Scale (PCS), ${ }^{28}$ the State-Trait Anxiety Inventory (STAI), ${ }^{29}$ and the Interpersonal Reactivity Index (IRI). ${ }^{30}$

The PCS is a 13-item measure of catastrophizing thoughts that includes three subscales: Rumination (the inability to stop thoughts concerning pain), Magnification (the tendency to exaggerate the threat value of pain stimuli), and Helplessness (the feeling of inability to deal with pain). The reliability of the Chinese version of the PCS (Cronbach's alpha $=0.91, \mathrm{~N}=153$ ) has been well established. ${ }^{28}$ The STAI (40 items comprising two subscales, ie, STAI-S and STAI$\mathrm{T})$ was adopted to assess the anxiety state and anxiety trait, with high reliabilities of its subscales (Cronbach's alpha $=0.90$ for STAI-S, Cronbach's alpha=0.80 for STAI-T, $\mathrm{N}=2150) .{ }^{29}$ Dispositional empathy was measured with the Chinese version of the IRI, which consists of 22 items comprising four subscales: Perspective Taking (the tendency to adopt other people's point of view), Fantasy Score (the tendency to transpose oneself imaginatively into the feelings and actions of fictitious characters in books, movies, and plays), Empathic Concern (the tendency to experience feelings of warmth, compassion, and concern for others), and Personal Distress (the tendency to experience the feelings of personal anxiety, discomfort, and unease in reaction to others' emotions). The Chinese version of the IRI has good internal consistency (Cronbach's alpha $=0.751, \mathrm{~N}=529) .{ }^{30}$ In the current sample, reliabilities of the scales were satisfactory: coefficient alphas were 0.936 for PCS, 0.913 for STAI-S, 0.890 for STAI-T, and 0.745 for IRI.

\section{Statistical analysis}

To rule out the possible difference of participants' characteristics, we performed one-way analysis of variance (ANOVA) on (1) age, (2) catastrophizing, (3) dispositional empathy, (4) anxiety state, and (5) anxiety trait among participants in the four experimental groups.

To ensure that the manipulation of "Effectiveness" in first-person experience groups (Groups 1 and 3) was successful, we performed two-way mixed-design ANOVA with "Cue" (low pain cues and high pain cues) as within-subject factor and "Effectiveness" (effective and ineffective) as between-subject factor. Meanwhile, to ensure that the manipulation of conditioning procedure in all groups was successful, we performed three-way mixed-design ANOVA, with "Cue" (low pain cues and high pain cues) as withinsubject factor, "Acquisition Way" (experience in person and through observation) and "Effectiveness" as betweensubject factors.

To assess the influence of visual cues, experience acquisition ways, and effectiveness on pain ratings during the two tests, we performed three-way mixed-design ANOVA, with "Cue" (low pain cue, control cue, and high pain cue) as within-subject factor, "Acquisition Way" and "Effectiveness" as between-subject factors. The Huynh-Feldt correction was applied in light of observed violations of sphericity assumption, ${ }^{31}$ and the uncorrected degrees of freedom were reported for the convenience of the readers. Post hoc Bonferroni corrections were performed to account for multiple comparisons, where necessary.

We defined the difference between low pain cueassociated and control cue-associated ratings as placebo effect, and the difference between high pain cue-associated and control cue-associated ratings as nocebo effect. To verify the persistence of the placebo and nocebo effects, we performed three-way mixed-design ANOVA, with "Test" (test 1 and test 2) as a within-subject factor, and "Acquisition Way" and "Effectiveness" as between-subject factors.

To verify the difference between the placebo effect and nocebo effect, we calculated the mean placebo response and nocebo response across all four experimental groups for test 1 and test 2 , respectively. The mean values of placebo response and nocebo response were compared using paired-samples $t$-tests for each test separately.

All statistical analyses were carried out in SPSS 17.0 (SPSS Inc., New York, USA), and the statistical significance level was set at 0.05 . The effect size for ANOVA results in the present sample was estimated by partial eta-squared. For partial eta-squared, an effect size of 0.0099 is deemed as a "small" effect, around 0.0588 as a "medium" effect, and 0.1379 to infinity as a "large" effect. ${ }^{32}$ 


\section{Results}

\section{Participants' characteristics}

Participants' characteristics in each experimental group are summarized in Table 1. One-way ANOVA showed that there was no significant difference among the four experimental groups with regard to age and psychological characteristics (all $P>0.33$ ).

\section{The experimental manipulation during treatment and conditioning phases}

Two-way mixed-design ANOVA revealed a significant main effect of "Cue" on pain ratings $(\mathrm{F}(1,40)=880.589$, $\left.P<0.001, \eta_{\mathrm{p}}{ }^{2}=0.957\right)$. In addition, the interaction between "Effectiveness" and "Cue" was significant $\left(\mathrm{F}(1,40)=773.991, P<0.001, \eta_{\mathrm{p}}{ }^{2}=0.951\right)$. Post hoc tests showed that the high pain cue-associated ratings were higher than low pain cue-associated ratings $(P<0.001)$ in the effective condition. In contrast, there was no significant difference between high pain cue-associated ratings and low pain cue-associated ratings $(P>0.05)$ in the ineffective condition. However, the main effect of "Effectiveness" $\left(\mathrm{F}(1,40)=0.781, P=0.382, \eta_{\mathrm{p}}{ }^{2}=0.019\right)$ was not significant. These results indicated that the manipulation of "Effectiveness" in first-person experience groups was successful during the treatment phase, that is, it was "effective" for Group 1 but "ineffective" for Group 3.

Meanwhile, three-way mixed-design ANOVA revealed significant main effects of "Cue" $(F(1,78)=6576.055$, $\left.P<0.001, \eta_{\mathrm{p}}{ }^{2}=0.988\right)$ and "Effectiveness" $(\mathrm{F}(1,78)=7.042$, $\left.P=0.010, \eta_{\mathrm{p}}{ }^{2}=0.083\right)$ on pain ratings during the conditioning phase. Specifically, the high pain cue-associated ratings were higher than low pain cue-associated ratings $(P<0.001)$, and the ratings in the effective condition were higher than those in the ineffective condition $(P=0.010)$. However, the main effect of "Acquisition Way" $(\mathrm{F}(1,78)=0.001$, $\left.P=0.978, \eta_{\mathrm{p}}{ }^{2}<0.001\right)$ was not significant. In addition, the interaction between "Effectiveness" and "Cue" $(\mathrm{F}(1$, 78) $\left.=2.903, P=0.092, \eta_{\mathrm{p}}{ }^{2}=0.036\right)$, the interaction between "Cue" and "Acquisition Way" $(\mathrm{F}(1,78)=2.176, P=0.144$, $\left.\eta_{\mathrm{p}}{ }^{2}=0.027\right)$, the interaction between "Effectiveness" and "Acquisition Way" $\left(\mathrm{F}(1,78)=0.238, P=0.627, \eta_{\mathrm{p}}{ }^{2}=0.003\right)$, and the interaction among "Cue", "Effectiveness", and "Acquisition Way" $\left(\mathrm{F}(1,78)=0.016, P=0.899, \eta_{\mathrm{p}}{ }^{2}<0.001\right)$ were not significant during the conditioning phase. These results indicated that the manipulation of conditioning was successful in all groups.

\section{Placebo and nocebo responses in tests I and 2 \\ Test I}

Three-way mixed-design ANOVA revealed a significant main effect of "Cue" on pain ratings $(\mathrm{F}(2,156)=282.833$, $\left.P<0.001, \eta_{\mathrm{p}}{ }^{2}=0.784\right)$. Post hoc tests showed that the low pain cue-associated ratings were significantly lower than the control cue-associated ratings (Group 1: $P<0.001$; Group 2: $P<0.001$; Group 3: $P=0.010$; Group 4: $P=0.008$ ), and the high pain cue-associated ratings were significantly higher than the control cue-associated ratings (Group 1: $P<0.001$; Group 2: $P<0.001$; Group 3: $P<0.001$; Group 4: $P=0.001$ ), indicating that placebo responses and nocebo responses were consistently elicited in all experimental groups during test 1 (see Figure 2 upper panel). In addition, the interaction between "Effectiveness" and "Cue" was significant $(\mathrm{F}(2$, 156) $=54.552, P<0.001, \eta_{\mathrm{p}}{ }^{2}=0.412$ ). Post hoc tests showed that the low pain cue-associated ratings in the effective condition were lower than those in the ineffective condition $(P<0.001)$, and the high pain cue-associated ratings in the effective condition were higher than those in the ineffective condition $(P<0.001)$ (see Figure 3 left panel).

However, the main effects of "Acquisition Way" $\left(\mathrm{F}(1,78)=0.037, P<0.848, \eta_{\mathrm{p}}{ }^{2}<0.001\right)$ and "Effectiveness" $\left(\mathrm{F}(1,78)=1.800, P=0.184, \eta_{\mathrm{p}}{ }^{2}=0.023\right)$ were not significant. In addition, the interaction between "Cue" and "Acquisition Way" $\left(\mathrm{F}(2,156)=0.040, P=0.993, \eta_{\mathrm{p}}{ }^{2}=0.001\right)$, the interaction between "Effectiveness" and "Acquisition Way" $\left(\mathrm{F}(1,78)=0.298, P=0.587, \eta_{\mathrm{p}}{ }^{2}=0.004\right)$, and the interaction among "Cue," "Effectiveness," and "Acquisition Way" $\left(\mathrm{F}(2,156)=0.644, P=0.493, \eta_{\mathrm{p}}{ }^{2}=0.008\right)$ were not significant.

\section{Test 2}

Similar to the results in test 1 , three-way mixed-design ANOVA revealed a significant main effect of "Cue" on pain ratings $\left(\mathrm{F}(2,156)=164.406, P<0.001, \eta_{\mathrm{p}}{ }^{2}=0.678\right)$. Post hoc tests showed that the low pain cue-associated ratings were significantly lower than the control cue-associated ratings in Group $1(P<0.001)$, Group $2(P<0.001)$, and Group 3 $(P=0.027)$, but not in Group $4(P=0.224)$. In contrast, the high pain cue-associated ratings were significantly higher than the control cue-associated ratings in all experimental groups (Group 1: $P<0.001$; Group 2: $P<0.001$; Group 3: $P=0.033$; Group 4: $P=0.006$ ) (see Figure 2 lower panel). The interaction between "Effectiveness" and "Cue" was significant $\left(\mathrm{F}(2,156)=47.454, P<0.001, \eta_{\mathrm{p}}{ }^{2}=0.378\right)$. Post hoc tests showed that the low pain cue-associated ratings 


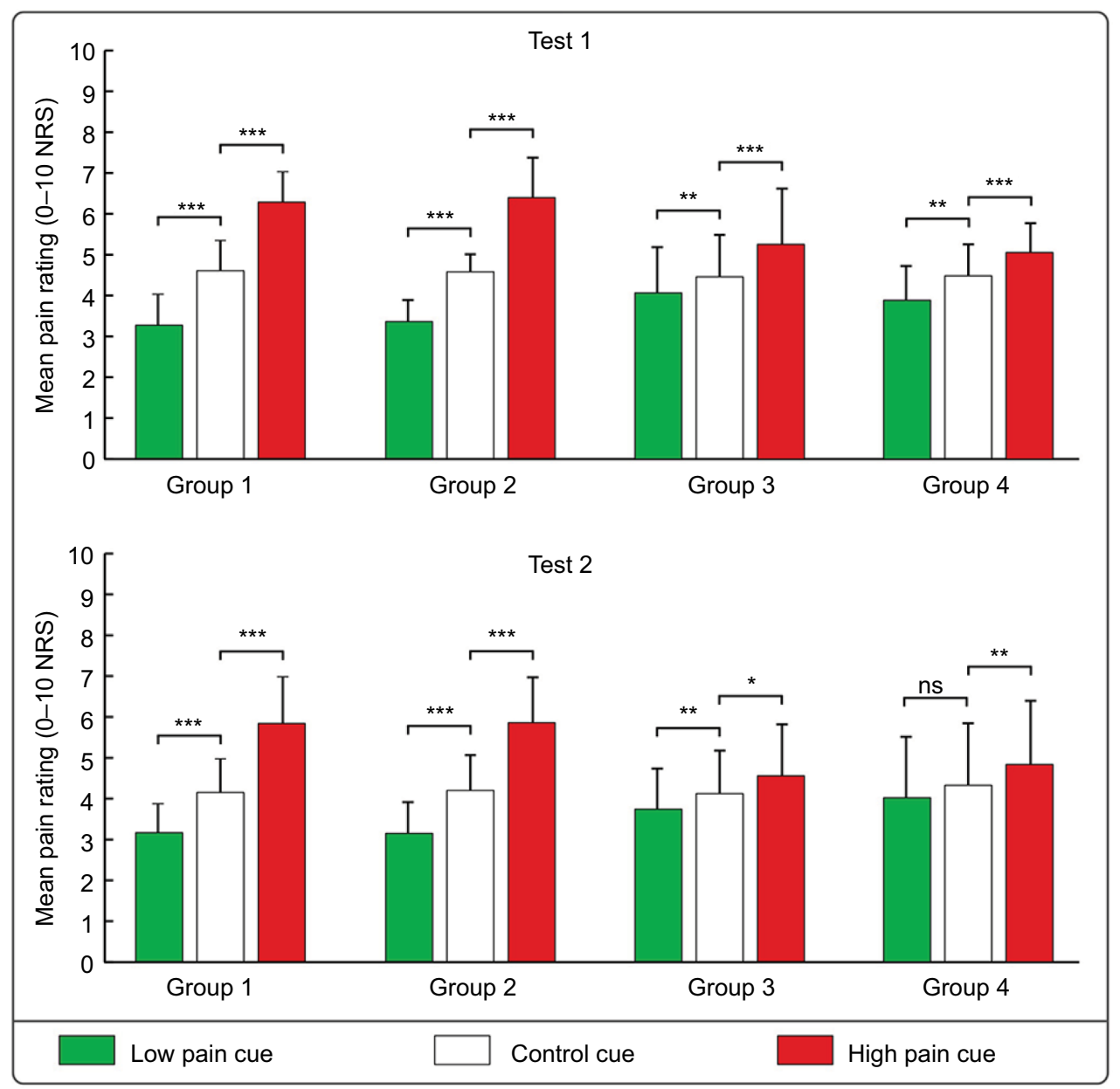

Figure 2 The influence of visual cues, experience acquisition ways, and effectiveness on pain ratings.

Notes: The histograms show that mean pain ratings to the nociceptive stimuli followed the low pain cues, the control cues, and the high pain cues, in each experimental group during test I (upper panel) and test 2 (lower panel). The low pain cue-associated ratings were significantly lower than the control cue-associated ratings, and the high pain cue-associated ratings were significantly higher than the control cue-associated ratings for each group both in tests I and 2 , except for $G$ roup 4 in test 2 . $* P<0.05$; $* * P<0.0$ I; ***P<0.001.

Abbreviations: NRS, Numeric Rating Scale; ns, not significant.

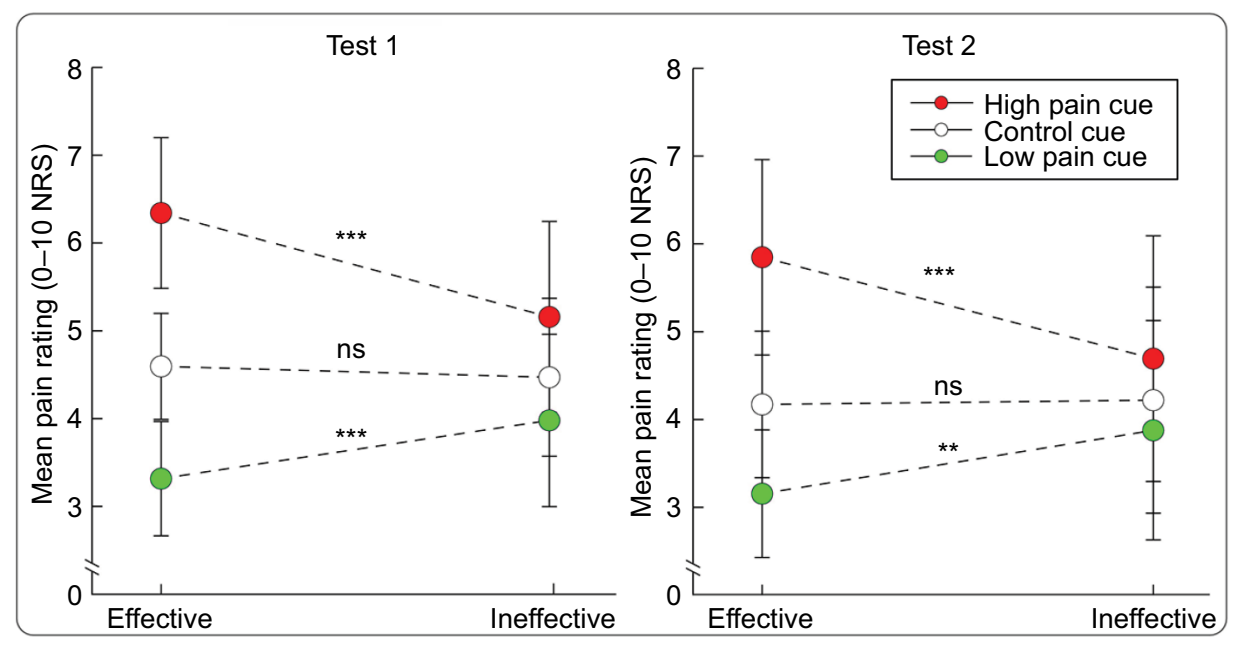

Figure 3 The significant interaction between "Effectiveness" and "Cue" on pain ratings.

Notes: Significant interactions between "Effectiveness" and "Cue" were observed in both tests I and 2. **P<0.0I; $* * * P<0.00$ I.

Abbreviations: NRS, Numeric Rating Scale; ns, not significant. 
in the effective condition were lower than those in the ineffective condition $(P=0.002)$, and the high pain cueassociated ratings in the effective condition were higher than those in the ineffective condition $(P<0.001)$ (see Figure 3 right panel).

However, the main effects of "Acquisition Way" ( $\mathrm{F}(1$, $\left.78)=0.362, P<0.549, \eta_{\mathrm{p}}{ }^{2}<0.005\right)$ and "Effectiveness" ( $\mathrm{F}(1$, $\left.78)=0.318, P=0.575, \eta_{\mathrm{p}}{ }^{2}=0.004\right)$ were not significant. The interaction between "Cue" and "Acquisition Way" (F(2, 156) $\left.=0.007, P=0.985, \eta_{\mathrm{p}}{ }^{2}<0.001\right)$, the interaction between "Acquisition Way" and "Effectiveness" $(\mathrm{F}(1,78)=0.285$, $\left.P=0.595, \eta_{\mathrm{p}}{ }^{2}=0.004\right)$, and the interaction among "Cue," "Acquisition Way," and "Effectiveness" $(\mathrm{F}(2,156)=0.073$, $P=0.898, \eta_{\mathrm{p}}{ }^{2}=0.001$ ) were not significant.

\section{The influence of prior experience on placebo and nocebo responses}

\section{Placebo response}

Three-way mixed-design ANOVA revealed significant main effects of "Effectiveness" $(\mathrm{F}(1,78)=33.633, P<0.001$, $\left.\eta_{\mathrm{p}}{ }^{2}=0.301\right)$ and "Test" $\left(\mathrm{F}(1,78)=7.522, P=0.008, \eta_{\mathrm{p}}{ }^{2}=0.088\right)$ on the placebo response, that is, the difference between low pain cue-associated and control cue-associated ratings. In contrast, the main effect of "Acquisition Way" ( $F(1$, $\left.78)=0.027, P=0.871, \eta_{p}{ }^{2}<0.001\right)$, the interaction between "Acquisition Way" and "Effectiveness" $(\mathrm{F}(1,78)=0.132$, $\left.P=0.717, \eta_{\mathrm{p}}{ }^{2}=0.002\right)$, the interaction between "Acquisition Way" and "Test" $\left(\mathrm{F}(1,78)=0.098, P=0.755, \eta_{\mathrm{p}}{ }^{2}=0.001\right)$, the interaction between "Effectiveness" and "Test" ( F (1, $\left.78)=0.512, P=0.476, \eta_{\mathrm{p}}{ }^{2}=0.007\right)$, and the interaction among "Acquisition Way," "Effectiveness," and "Test" (F(1, $78)=2.442, P=0.122, \eta_{\mathrm{p}}{ }^{2}=0.030$ ) were not significant. These results indicated that the placebo response in the effective condition was significantly larger than that in the ineffective condition regardless of the way to acquire the prior experience, and the placebo response in test 2 was significantly smaller than that in test 1 (see Figure 4 left panel), indicating that the placebo response was easy to be extinguished.

\section{Nocebo response}

Three-way mixed-design ANOVA revealed a significant main effect of "Effectiveness" $(\mathrm{F}(1,78)=56.165$, $\left.P<0.001, \eta_{\mathrm{p}}{ }^{2}=0.419\right)$ on the nocebo response, that is, the difference between high pain cue-associated and control cue-associated ratings. In contrast, the main effects of "Acquisition Way" $\left(\mathrm{F}(1,78)=0.005, P=0.944, \eta_{\mathrm{p}}{ }^{2}<0.001\right)$ and "Test" $\left(\mathrm{F}(1,78)=2.909, P=0.092, \eta_{\mathrm{p}}{ }^{2}=0.036\right)$, as well as the interaction between "Acquisition Way" and "Effectiveness" $\left(\mathrm{F}(1,78)=0.187, P=0.666, \eta_{\mathrm{p}}{ }^{2}=0.002\right)$, the interaction between "Acquisition Way" and "Test" $(\mathrm{F}(1,78)=0.148$, $\left.P=0.702, \eta_{\mathrm{p}}{ }^{2}=0.002\right)$, the interaction between "Effectiveness" and "Test" $\left(\mathrm{F}(1,78)=0.693, P=0.408, \eta_{\mathrm{p}}{ }^{2}=0.009\right)$, and the interaction among "Acquisition Way," "Effectiveness," and "Test" $\left(\mathrm{F}(1,78)=2.050, P=0.156, \eta_{\mathrm{p}}{ }^{2}=0.026\right)$ were not significant. These results indicated that the nocebo response in the effective condition was significantly more profound than that in the ineffective condition regardless of the way to acquire the prior experience. Different from the placebo effect, no significant difference of the nocebo response was observed between tests 1 and 2 (see Figure 4 right panel).

\section{The comparison between placebo and nocebo responses}

As can be seen in Figure 5, the nocebo response was significantly larger than the placebo response in both test 1 $(\mathrm{t}(81)=-3.23 ; P=0.002$, two-tailed $)$ and test $2(\mathrm{t}(81)=-3.44$;

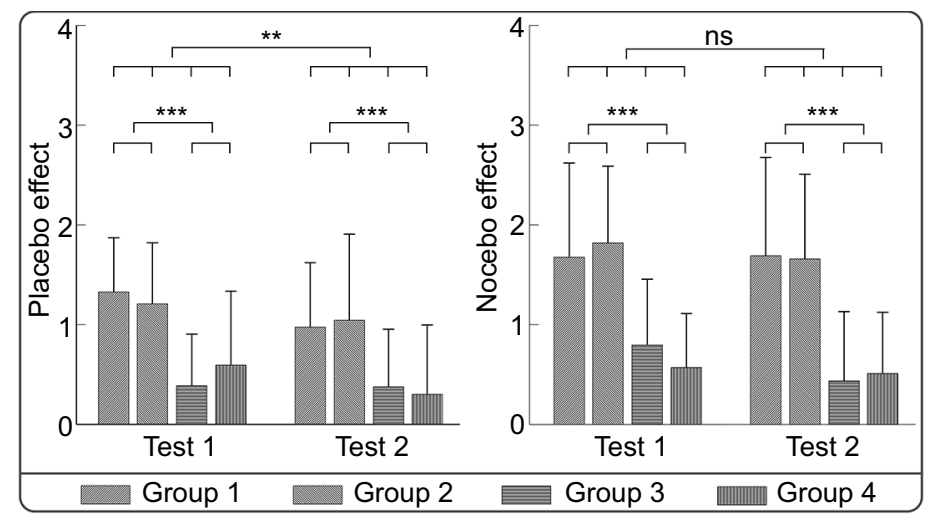

Figure 4 The influence of prior experience on placebo and nocebo responses.

Notes: The placebo response (the difference between low pain cue-associated and control cue-associated ratings; left panel) and the nocebo response (the difference between high pain cue-associated and control cue-associated ratings; right panel) were displayed for each experimental group in tests $I$ and 2 . $* * P<0.0 I$; $* * * P<0.00 I$. Abbreviation: ns, not significant. 


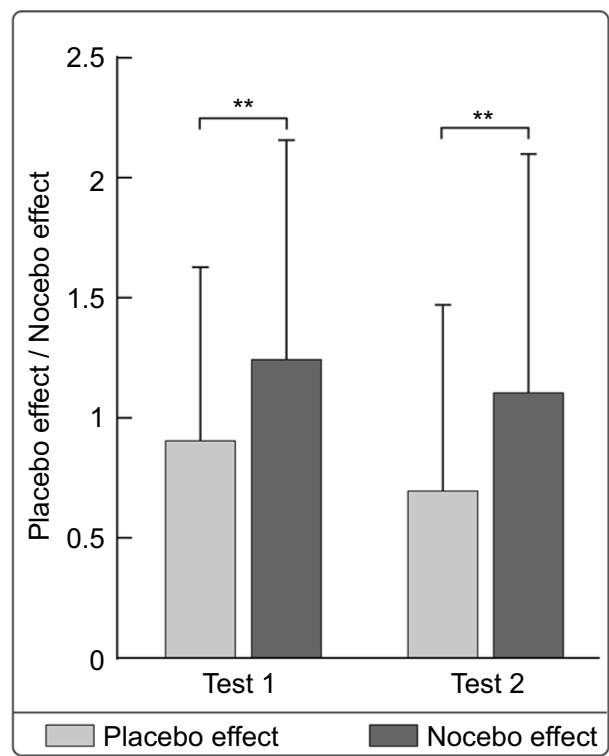

Figure 5 The comparison of placebo and nocebo responses.

Notes: The nocebo response was significantly larger than the placebo response across all experimental groups both in tests I and 2 . ${ }^{*} * P<0.0 \mathrm{I}$.

$P=0.001$, two-tailed), indicating that our experimental design had a greater effect on the expectancy of aversive events.

\section{Discussion}

There were two salient observations from the present results. First, experience gained 5-6 days ago in person and through social observation both can affect placebo and nocebo effects to subsequent treatments, and the response magnitudes of the two experience acquisition ways were comparable. In other words, both placebo and nocebo responses in the effective condition were significantly larger than those in the ineffective condition, regardless of the acquisition ways of prior experience. Second, once placebo and nocebo effects were elicited, nocebo effect was likely to persist, while placebo effect was easy to diminish.

\section{The sustainability of placebo and nocebo effects to subsequent treatments}

Both first-person and social observation prior experience obtained a while ago (5-6 days in our case) can affect placebo and nocebo responses to subsequent treatments, with no difference in the magnitudes of the placebo and nocebo responses between two acquisition ways.

One possible explanation is that experience obtained through social observation has comparable potentials as first-person experience in affecting perceived effectiveness of treatments. Similar to the first-person experience, experience obtained by social observation can increase or decrease expec- tancies on analgesia and hyperalgesia effects, ${ }^{12}$ depending on what has been observed. Given its extremely essential role in survival, analgesia and hyperalgesia information tends to be consolidated into long-term memory. ${ }^{33}$ Therefore, when the observer undergoes a similar circumstance even after several days, analgesia and hyperalgesia information can be recalled easily to facilitate the processes of potentially rewarding events and harmful events, respectively. This finding provides further evidence to support Bandura's social cognitive learning theory, ${ }^{3}$ which emphasizes that the learning process initiated by social observation not only includes recognition of observed information, but also involves encoding and storage of this information in an easily remembered form to shape observer's experience. ${ }^{3,34}$ Indeed, when the observer undergoes a similar condition after observing a demonstrator receiving analgesic and hyperalgesic treatments, the retentive information can be easily extracted to rehearse the demonstrator's responses (eg, placebo analgesia/nocebo hyperalgesia). ${ }^{15}$ The neural mechanisms of placebo analgesia and nocebo hyperalgesia induced by prior experience through social observation might rely on the existence of the mirror neuron system responsible for the processing and storage of outcomes learned by observing others..$^{35}$ Alternatively, it is also possible that first-person and social observational experience obtained 5-6 days ago affect perceived effectiveness of treatments differently. The absence of a difference as a function of "Acquisition Way" might be due to the conditioning phase prior to the test phase, which may interact with the prior experience, regardless of how it was gained, thereby resulting in comparable placebo and nocebo responses.

In addition, prior experience obtained via effective conditioning procedure induced significantly larger placebo and nocebo responses to subsequent treatments in comparison to those obtained via ineffective conditioning procedure, regardless of the acquisition ways (ie, experience in person or through observation). This finding is in line with previous studies focusing on how effectiveness of prior experience in person affects placebo responses, ${ }^{12,36}$ and consistent with the study of Kessner et al, ${ }^{13,37}$ which indicated that treatmentrelated experience critically determines the response to the second, different analgesic treatment.

There might be two distinct mechanisms underlying the effectiveness effect. One may rely on the modification on response expectancy. As suggested by response expectancy theory, personal responses could be altered by changing individuals' expectancy. ${ }^{8,38-41}$ It has been suggested that positive expectancy plays an important role in triggering the release of endogenous opioids and then produces placebo analgesia, ${ }^{42}$ 
whereas expecting a negative outcome (hyperalgesia) may trigger the release of cholecystokinin, which in turn produces nocebo hyperalgesia. ${ }^{8,43}$ Specifically, high expectancy level could enhance placebo and nocebo responses to the treatment of pain, while relative low expectancy level could weaken such responses. ${ }^{44-46}$ In line with the response expectancy theory, effective prior experience, gained in person or through observation, might be associated with increased expectancy, leading to substantial placebo and nocebo responses. In contrast, ineffective prior experience could not enhance the level of expectancy, therefore generating comparatively weak placebo and nocebo responses. Consistent with this theory, a previous neuroimaging study showed that a strong placebo induced better analgesic efficacy than a weak placebo, and the response in rostral anterior cingulate cortex was significantly higher to the strong placebo than to the weak placebo. ${ }^{47}$ It indicated that the efficacy of placebo treatments depended on participants' expectancy, which was, at least partly, shaped by previous experiences. The efficacy of placebo treatments may rely on the learning process, as the influence of treatment history could transfer over time and over therapeutic approaches. ${ }^{37}$ Zunhammer et al found that the negative carry-over effects on treatment efficacy were not counteracted by the expectations of positive treatment effects (induced by changing the drug administration way) in the subjects, which indicated that learned carry-over effects generalize over time and across routes of drug administration regardless of conscious expectations. ${ }^{48}$

\section{The persistence of placebo and nocebo effects}

We observed a reduced placebo response in test 2 compared with that in test 1 , whereas nocebo response measured within the two tests were not discriminable, indicating that nocebo effect is more likely to persist at least in the two tests. Differences in the persistence of placebo and nocebo effects might be associated with the strength of learning. ${ }^{19}$ On the other hand, given that the test phase can be considered as an extinction process (extinction process is also one kind of learning process), ${ }^{12}$ the observed differences in terms of the persistence of the placebo and nocebo effects might result from their differences in extinction processes. This finding is consistent with previous studies suggesting that nocebo hyperalgesia is difficult to be extinguished once established via conditioning procedure. ${ }^{20,21}$ Furthermore, the nocebo response was more pronounced than the placebo response across all four experimental groups in the two tests, and a similar finding has been reported in a previous study. ${ }^{49}$ From an evolutionary standpoint, threat-related signals are more salient than safety-related signals for detecting potential changes of the environment. ${ }^{50-53}$ Nocebo responses, therefore, represent a valuable adaptation to enhance the perceptual processing and anticipation of negative outcomes in response to the challenges in the environment, which facilitates the initiation of potentially defensive behaviors. ${ }^{54}$ That is, once the acquisition of nocebo effect succeeded, its natural extinction processes slowly, eventually resulting in the maintenance of the information over time.

\section{Study limitations}

Several research questions related to this study need to be addressed in further investigations. For instance, only right-handed female participants were enrolled, and this may restrict the generalization of the experimental results. Moreover, expectancy and state anxiety were not assessed, therefore limiting our understanding about the weight of participants' expectancy and state anxiety on placebo/nocebo effects. In addition, it may require an additional group with no prior experience as a "baseline" to reveal either prior exposure to an effective treatment enhanced the following conditioning effect, or prior exposure to an ineffective treatment muted it.

\section{Clinical implications}

Our observations have two important clinical implications. First, our study highlights that the influences of prior experience gained through social observation on the placebo/ nocebo response are similar to those of prior experience obtained in person, and that the effective condition induced more pronounced placebo/nocebo responses than the ineffective condition. Therefore, it is important for clinical practice to increase positive prior experience generating placebo analgesia and to decrease negative prior experience producing nocebo hyperalgesia, ${ }^{55}$ through not only first-person experience, but also social observation. Enhanced placebo effect and/or reduced nocebo effect would promote patients' rehabilitation by optimizing their treatment expectancy.

Second, it is worth noting that the nocebo response was more significant than the placebo response, and nocebo response is more likely to persist. Thus, the negative prior experience generating nocebo hyperalgesia should be emphasized in clinical applications, as it is likely to trigger a undesirable response to subsequent treatments, which would increase the severity of symptoms immediately and persistently. For instance, doctors may need to mind their behaviors and attitudes toward patients, given that negative verbal suggestions can lead to unimaginable impairments for 
patients' health. ${ }^{56}$ Attention to the extinction of the existed experience with negative responses (eg, nocebo hyperalgesia) should be warranted in the future.

\section{Acknowledgments}

This work was supported by the National Natural Science Foundation of China (Nos. 31471082, 31671141, 31701000), Chongqing Research Program of Basic Research and Frontier Technology (No. cstc2015jcyjBX0050), and the Scientific Foundation project of Institute of Psychology, Chinese Academy of Sciences (Nos. Y6CX021008, Y6CX281007). The funders had no role in study design, data collection and analysis, decision to publish, or preparation of the manuscript.

\section{Disclosure}

The authors report no conflicts of interest in this work.

\section{References}

1. Colloca L. Placebo, nocebo, and learning mechanisms. Handb Exp Pharmacol. 2014;225:17-35.

2. Colloca L, Sigaudo M, Benedetti F. The role of learning in nocebo and placebo effects. Pain. 2008;136(1):211-218.

3. Bandura A. Social Learning Theory. Englewood Cliffs, NJ: PrenticeHall; 1977:459-467.

4. Kantor TG, Sunshine A, Laska E, Meisner M, Hopper M. Oral analgesic studies: pentazocine hydrochloride, codeine, aspirin, and placebo and their influence on response to placebo. Clin Pharmacol Ther. 1966;7(4):447-454.

5. Laska E, Sunshine A. Anticipation of analgesia. A placebo effect. Headache. 1973;13(1):1-11.

6. Lasagna L, Mosteller F, Von Felsinger JM, Beecher HK. A study of the placebo response. Am J Med. 1954;16:770-779.

7. Goebel MU, Trebst AE, Steiner J, et al. Behavioral conditioning of immunosuppression is possible in humans. FASEB J. 2002;16(6):1869-1873.

8. Benedetti F, Pollo A, Lopiano L, Lanotte M, Vighetti S, Rainero I. Conscious expectation and unconscious conditioning in analgesic, motor, and hormonal placebo/nocebo responses. J Neurosci. 2003;23(10):4315-4323.

9. Doering BK, Rief W. Utilizing placebo mechanisms for dose reduction in pharmacotherapy. Trends Pharmacol Sci. 2012;33(3):165-172.

10. Planes S, Villier C, Mallaret M. The nocebo effect of drugs. Pharmacol Res Perspect. 2016;4(2):e00208.

11. Bavbek S, Aydin O, Sozener ZC, Yuksel S. Determinants of nocebo effect during oral drug provocation tests. Allergol Immunopathol(Madr.). 2015;43(4):339-345.

12. Colloca L, Benedetti F. How prior experience shapes placebo analgesia. Pain. 2006;124(1-2):126-133.

13. Kessner S, Wiech K, Forkmann K, Ploner M, Bingel U. The effect of treatment history on therapeutic outcome: an experimental approach. JAMA Intern Med. 2013;173(15):1468-1469.

14. Colloca L, Benedetti F. Placebo analgesia induced by social observational learning. Pain. 2009;144(1-2):28-34.

15. Hunter T, Siess F, Colloca L. Socially induced placebo analgesia: a comparison of a pre-recorded versus live face-to-face observation. Eur J Pain. 2014;18(7):914-922.

16. Swider K, Babel P. The effect of the type and colour of placebo stimuli on placebo effects induced by observational learning. PLoS One. 2016;11(6): $\mathrm{e} 0158363$.

17. Swider K, Babel P. The effect of the sex of a model on nocebo hyperalgesia induced by social observational learning. Pain. 2013;154(8):1312-1317.
18. Vogtle E, Barke A, Kroner-Herwig B. Nocebo hyperalgesia induced by social observational learning. Pain. 2013;154(8):1427-1433.

19. Colloca L, Petrovic P, Wager TD, Ingvar M, Benedetti F. How the number of learning trials affects placebo and nocebo responses. Pain. 2010;151(2):430-439.

20. Au Yeung ST, Colagiuri B, Lovibond PF, Colloca L. Partial reinforcement, extinction, and placebo analgesia. Pain. 2014;155(6):1110-1117.

21. Colagiuri B, Quinn VF, Colloca L. Nocebo hyperalgesia, partial reinforcement, and extinction. J Pain. 2015;16(10):995-1004.

22. Pud D, Golan Y, Pesta R. Hand dominancy-a feature affecting sensitivity to pain. Neurosci Lett. 2009;467(3):237-240.

23. Inui K, Tran TD, Hoshiyama M, Kakigi R. Preferential stimulation of Adelta fibers by intra-epidermal needle electrode in humans. Pain. 2002;96(3):247-252.

24. Kakigi R, Inui K, Tamura Y. Electrophysiological studies on human pain perception. Clin Neurophysiol. 2005;116(4):743-763.

25. Gracely RN. Studies of pain in normal man. In: Wall PD, Melzack R, editors. The Textbook of Pain. 3rd ed. New York: ChurchillLivingstone. 1994:315-336.

26. von Baeyer CL. Numerical rating scale for self-report of pain intensity in children and adolescents: recent progress and further questions. Eur J Pain. 2009;13(10):1005-1007.

27. Craen AJMD. Effect of colour of drugs: systematic review of perceived effect of drugs and of their effectiveness. BMJ. 1996;313(7072):1624-1626.

28. Xu X, Wei X, Wang F, et al. Validation of a simplified Chinese version of the pain catastrophizing scale and an exploration of the factors predicting catastrophizing in pain clinic patients. Pain Phys. 2015;18(6):e1059-31072.

29. Shek DTL. Reliability and factorial structure of the Chinese version of the State-Trait Anxiety Inventory. J Psychopathol Behav Assess. 1988;10(4):303-317.

30. Zhang FF, Dong Y, Wang K. Reliability and validity of the Chinese version of the interpersonal reactivity index-C. Chin J Clin Psychol. 2010;18(2):155-157.

31. Girden ER. ANOVA: repeated measures. J Marketing Res. 1995;35:60-61.

32. Cohen J. Statistical Power Analysis for the Social Sciences. Burlington: Elsevier; Science; 1988

33. Marchand S. The Phenomenon of Pain. Seattle, USA: IASP; 2012:9-10.

34. Bandura A. Chapter 3. Modeling theory: some traditions, trends, and disputes 1. Recent Trends in Social Learning Theory. New York: Academic Press; 1972:35-61.

35. Iacoboni M. Imitation, empathy, and mirror neurons. Annu Rev Psychol. 2009;60:653-670.

36. Schafer SM, Colloca L, Wager TD. Conditioned placebo analgesia persists when subjects know they are receiving a placebo. J Pain. 2015;16(5):412-420.

37. Kessner S, Forkmann K, Ritter C, Wiech K, Ploner M, Bingel U. The effect of treatment history on therapeutic outcome: psychological and neurobiological underpinnings. PLoS One. 2014;9(10):e109014.

38. Bingel U, Wanigasekera $\mathrm{V}$, Wiech $\mathrm{K}$, et al. The effect of treatment expectation on drug efficacy: imaging the analgesic benefit of the opioid remifentanil. Sci Transl Med. 2011;3(70):70ra14.

39. Koyama T, McHaffie JG, Laurienti PJ, Coghill RC. The subjective experience of pain: where expectations become reality. Proc Natl Acad Sci. 2005;102(36):12950-12955.

40. Wager TD, Rilling JK, Smith EE, et al. Placebo-induced changes in FMRI in the anticipation and experience of pain. Science. 2004;303(5661):1162-1167.

41. Montgomery GH, Kirsch I. Classical conditioning and the placebo effect. Pain. 1997;72(1):107-113.

42. Amanzio M, Benedetti F. Neuropharmacological dissection of placebo analgesia: expectation-activated opioid systems versus conditioningactivated specific subsystems. J Neurosci. 1999;19(1):484-494.

43. Benedetti F, Amanzio M, Casadio C, Oliaro A, Maggi G. Blockade of nocebo hyperalgesia by the cholecystokinin antagonist proglumide. Pain. 1997;71(2):135-140. 
44. Colloca L, Lopiano L, Lanotte M, Benedetti F. Overt versus covert treatment for pain, anxiety, and Parkinson;s disease. Lancet Neurol. 2004;3(11):679-684.

45. Enck P, Bingel U, Schedlowski M, Rief W. The placebo response in medicine: minimize, maximize or personalize? Nat Rev Drug Discov. 2013;12(3):191-204.

46. Pollo A, Amanzio M, Arslanian A, Casadio C, Maggi G, Benedetti F. Response expectancies in placebo analgesia and their clinical relevance. Pain. 2001;93(1):77-84.

47. Geuter S, Eippert F, Hindi Attar C, Buchel C. Cortical and subcortical responses to high and low effective placebo treatments. Neuroimage. 2013;67:227-236

48. Zunhammer M, Ploner M. The effects of treatment failure generalize across different routes of drug administration. Sci Transl Med. 2017;9(393):eaal2999.

49. Jensen K, Kirsch I, Odmalm S, Kaptchuk TJ, Ingvar M. Classical conditioning of analgesic and hyperalgesic pain responses without conscious awareness. Proc Natl Acad Sci. 2015;112(25):7863-7867.
50. Hansen $\mathrm{CH}$, Hansen RD. Finding the face in the crowd: an anger superiority effect. J Pers Soc Psychol. 1988;54(6):917-924.

51. Ohman A, Lundqvist D, Esteves F. The face in the crowd revisited: a threat advantage with schematic stimuli. J Pers Soc Psychol. 2001;80(3):381-396.

52. Rozin P, Royzman EB. Negativity bias, negativity dominance, and contagion. Pers Soc Psychol Rev. 2001;5(4):296-320.

53. Alpers GW, Ruhleder M, Walz N, Muhlberger A, Pauli P. Binocular rivalry between emotional and neutral stimuli: a validation using fear conditioning and EEG. Int J Psychophysiol. 2005;57(1):25-32.

54. Woody EZ, Szechtman H. Adaptation to potential threat: the evolution, neurobiology, and psychopathology of the security motivation system. Neurosci Biobehav Rev. 2011;35(4):1019-1033.

55. Klinger R, Blasini M, Schmitz J, Colloca L. Nocebo effects in clinical studies: hints for pain therapy. Pain Rep. 2017;2(2):e586.

56. Blasini M, Corsi N, Klinger R, Colloca L. Nocebo and pain: an overview of the psychoneurobiological mechanisms. Pain Reports. 2017;2(2):e585.
Journal of Pain Research

\section{Publish your work in this journal}

The Journal of Pain Research is an international, peer reviewed, open access, online journal that welcomes laboratory and clinical findings in the fields of pain research and the prevention and management of pain. Original research, reviews, symposium reports, hypothesis formation and commentaries are all considered for publication.

\section{Dovepress}

The manuscript management system is completely online and includes a very quick and fair peer-review system, which is all easy to use. Visit http://www.dovepress.com/testimonials.php to read real quotes from published authors. 